\title{
The Effect of New Diluents on Motility and Viability of Rabbit Spermatozoa
}

\author{
Martin Massanyi $^{1 *}$, Tomas Slanina $^{1}$, Lubomir Ondruska $^{2}$ and Peter Massanyi ${ }^{1}$ \\ 1. Slovak University of Agriculture in Nitra, Nitra, Slovak Republic \\ 2. Research Institute of Animal Production, Nitra, Slovak Republic \\ * Corresponding author: martinmassanyi@yahoo.com
}

The aim of the study was to evaluate the effect of new developed diluents [1,2] on rabbit spermatozoa motility and viability. Semen samples used in experiment were collected from New Zealand White rabbits. After processing, the semen samples were stored in the laboratory cooler at $5^{\circ} \mathrm{C}$. The main focus was to evaluate the basic parameters of rabbit spermatozoa motility in in vitro conditions using different new diluents (SMM1, SMM2, SMM3) concentrations at different time periods ( $0-5$ and 24 hours). The effect of selected diluents on rabbit spermatozoa motility parameters were determined using the CASA method using microscopic analysis [3]. The total spermatozoa motility in our experimental samples was in the range of $90.59-31.28 \%$ (SMM1), $85.94-28,15 \%$ (SMM2) and 89.48 - 24.66\% (SMM3) compared to control $(87.82-20.51 \%)$. No significant decrease in experimental samples at time periods of $0,1,2,3,4$, 5 hours neither after 24 hours compared to the basic levels were found. Percentage of progressively motile spermatozoa showed similar values in all experimental groups compared with control group. A significant stimulatory effect was noticed after 24 hours by significant decrease in motility. Likewise, decrease of distance and velocity parameters was detected in spermatozoa cultivated in vitro with the diluents. Similarly, the stimulating effect was found in the experimental samples for other parameters. Spermatozoa viability was verified using MTT assay in time intervals of $0,1,2,3,4,5$ and no significant differences compared with control were detected. Genetic analysis of spermatozoa was also realized with no DNA changes. The results of our work show positive effect of new diluents (SMM1, SMM2 and SMM3) on rabbit spermatozoa motility parameters.

\section{References:}

[1] T Slanina et al., Journal of Microbiology, Biotechnology and Food Sciences 1 (2012), p. 573.

[2] T Slanina et al., Acta Veterinaria Hungarica 66 (2018a), p. 135.

[3] T Slanina et al., Czech Journal of Animal Sciences 63 (2018b), p. 127.

[4] This work was supported by projects VEGA 1/0539/18, APVV-16-0289, and KEGA 010/SPU-4/2018. 